\title{
El consentimiento informado: su importancia para la investigación retrolectiva y el progreso de la ciencia médica
}

\section{Edith Valdez-Martínez ${ }^{*}$ y Miguel Bedolla²}

${ }^{1}$ Instituto Mexicano del Seguro Social, Coordinación Nacional de Investigación en Salud, Ciudad de México, México; ${ }^{2}$ Northwest Vista College of San Antonio, Departamento de Educación, Texas, Estados Unidos de América

\section{Resumen}

En la investigación retrolectiva, la información necesaria para responder la pregunta de investigación se genera directamente de expedientes clínicos y de otras fuentes clínico-documentales. Este artículo analiza la dispensa del consentimiento informado y el aviso de privacidad cuando la investigación es retrolectiva, de lo cual emergen dos líneas de argumentación: una es el deber del médico de proteger la dignidad, la integridad, el derecho a la autodeterminación, la intimidad del enfermo y la confidencialidad de la información obtenida de él; la otra es la contribución de las investigaciones retrolectivas al control de las enfermedades y a la mejora de la salud de la sociedad. La dispensa del consentimiento o el aviso de privacidad en el expediente clínico es importante para la investigación retrolectiva, pero tiene implicaciones éticas para los investigadores que no cumplan con la racionalidad y responsabilidad personal que tienen ante la sociedad.

PALABRAS CLAVE: Investigación retrolectiva. Consentimiento informado. Ética de la investigación. Comités de ética de la investigación.

\section{Informed consent: its importance for retrolective research and medical science progress}

\section{Abstract}

In retrolective research, the information necessary to answer the research question is directly generated from medical records and other clinical-documentary sources. This article analyzes the waiver of informed consent and privacy notice when research is retrolective, from which two lines of argument emerge: one is the physician's duty to protect patient dignity, integrity, right to self-determination and privacy, as well as the confidentiality of the information obtained from him; the other is retrolective research contribution to the control of diseases and society's health improvement. Waiver of informed consent or privacy notice documented in the medical record is important for retrolective research, but it has ethical implications for researchers who do not comply with the rationality and personal responsibility they have before society.

KEY WORDS: Retrolective research. Informed consent. Research ethics. Research ethics committees.

\section{Introducción}

En la atención médica de un enfermo, este otorga datos personales (e.g., nombre, domicilio, clave única de registro de población) y datos sensibles en relación con su historial médico y personal (e.g., origen étnico, creencias religiosas, estado de salud, ancestralidad, información genética, preferencias sexuales) ${ }^{1,2}$ Tanto los datos personales como los datos sensibles quedan registrados en el expediente
Correspondencia:

*Edith Valdez-Martínez

E-mail: edith.valdez@imss.gob.mx

DOl: 10.24875/GMM 20000227

DOI. 10.24875/GMM.20000227
Fecha de recepción: 24-04-2020

Fecha de aceptación: 15-09-2020

www.gacetamedicademexico.com CC BY-NC-ND (http://creativecommons.org/licenses/by-nc-nd/4.0/). 
clínico físico ${ }^{3}$ o electrónico, ${ }^{4}$ al cual tienen acceso irrestricto el médico y el equipo de salud. En el manejo de estos datos, el equipo de salud está obligado a guardar el secreto profesional para mantener la confidencialidad e intimidad de la información que el enfermo le ha confiado. ${ }^{1-4}$ Un investigador puede ser o no parte del equipo de salud y acceder a los expedientes clínicos con fines de investigación.

Las investigaciones retrolectivas ${ }^{5}$ emplean técnicas y métodos de investigación documental; más precisamente, la información se obtiene directamente de expedientes clínicos u otras fuentes clínico-documentales (e.g.: registros de cáncer, bancos de datos de anomalías genéticas, etcétera), producto de la relación médico-enfermo y que no fue planeada ex profeso para las necesidades de una investigación científica. Por lo tanto, el investigador se enfrenta con el problema de carecer del consentimiento informado de los potenciales sujetos participantes y el clínico afronta el riesgo de compartir un expediente clínico que contiene datos personales y sensibles protegidos por la ley. ${ }^{1,2}$

La controversia acerca de permitir o no al investigador el acceso a los datos personales sin el consentimiento de los potenciales participantes emerge de la consideración de dos vertientes: una es el deber del médico-profesional de la salud de proteger la dignidad, la integridad, el derecho a la autodeterminación, la intimidad del enfermo y la confidencialidad de la información personal que este le ha confiado; la otra es reconocer la contribución de las investigaciones retrolectivas a la búsqueda de soluciones a las enfermedades o problemas sanitarios y a la mejora de la salud de la sociedad.

La obligación de obtener consentimiento informado para acceder a datos del expediente clínico con fines de investigación retrolectiva es sometida en este escrito a un análisis crítico; se darán argumentos a favor y en contra considerando las leyes mexicanas vigentes y la práctica habitual. Las leyes mexicanas ${ }^{1,2}$ exigen consentimiento informado o dispensa de este cuando exista una previa disociación de los datos personales o la inclusión de un aviso de privacidad durante la atención médica. En la práctica habitual, el investigador accede a datos personales y sensibles de los enfermos sin aviso de privacidad y sin el consentimiento expreso del titular, es decir, del enfermo, a quien corresponde el contenido del expediente. Esta práctica la respalda el valor de la investigación retrolectiva (i.e., beneficio social, científico y clínico), las condiciones de confidencialidad en el manejo de datos personales descritas en el protocolo y el hecho de ser considerada como una investigación sin riesgo para el enfermo. ${ }^{6}$ Dos líneas de argumentación individuales pero complementarias, que tienen que ver con las doctrinas o principios de la no maleficencia y la justicia, subyacen bajo el poderoso mandamiento de respeto a los humanos.

\section{La no maleficencia}

La medicina moderna crea la obligación moral de abstenerse intencionalmente de realizar acciones que puedan causar daño e injuria; ${ }^{7}$ por ejemplo, el acceso (sin consentimiento) a datos personales y datos sensibles de los enfermos conlleva a vulnerar la dignidad, la integridad, el derecho a la autodeterminación, la intimidad y la confidencialidad de la información que el enfermo ha confiado únicamente para su atención clínica. Esta obligación moral pareciera imperar sobre la también poderosa obligación de beneficencia, la cual impone la obligación moral de ayudar a otros (los enfermos y la sociedad) a promover sus importantes y legítimos intereses. ${ }^{7}$ Para ilustrar: las enfermedades provocan aflicción, amenaza e incertidumbre a quienes las padecen, a sus familiares $y$, en general, a la sociedad. Esta aflicción e incertidumbre genera la necesidad de conocimiento válido que permita al médico explicar la enfermedad, y, a la par, implementar un tratamiento exitoso. La investigación en salud es, por lo tanto, un componente necesario para satisfacer esa necesidad.

No obstante, el respeto a la autonomía de los enfermos como potenciales sujetos de investigación establece límites a la mencionada obligación moral de beneficiar a otros, ${ }^{7}$ por ser los enfermos poseedores de los derechos de titularidad de la información contenida en el expediente clínico; pero, al mismo tiempo, este último planteamiento no puede ser considerado como un imperativo absoluto para impedir la investigación retrolectiva. Este tipo de investigación genera conocimiento fundamental para el descubrimiento, el desarrollo y la realización de intervenciones que se necesitan para la prevención y control de enfermedades específicas e, incluso, para mejorar la calidad de la atención que ofrecen los sistemas de salud. Además, provee información que es inaccesible a través de ensayos/estudios clínicos, los cuales requieren consentimiento informado por ser prospectivos y experimentales. Sin embargo, por un lado, la Declaración de Helsinki establece en la pauta $8: 8$ 
Aunque el objetivo principal de la investigación médica es generar nuevos conocimientos, este objetivo nunca debe tener primacía sobre los derechos y los intereses de la persona que participa en la investigación.

Esto le da más valor al bien de un individuo que al bien de muchos y sugiere que la investigación científica (aunque sea de reconocida calidad y garantice apropiadamente la confidencialidad) carece de superioridad absoluta. Por otra parte, el Council for International Organizations of Medical Sciences toma una posición a favor de la investigación, pues considera que un comité de ética de la investigación puede conceder la exención del consentimiento informado en investigación retrolectiva cuando esta no sea factible o viable sin dicha exención, cuando tenga un valor social importante y entrañe apenas riesgos mínimos para los participantes, aun cuando el estudio incluya datos identificables o muestras biológicas. ${ }^{9}$

Esta declaración refuerza la asunción cultural de que la investigación científica es primordial. Empero, ¿las evaluaciones de los riesgos pueden estar libres de presunciones basadas en valores? Al respecto, es importante resaltar que la ciencia señala los riesgos, pero son los valores de los investigadores (como humanos) los que determinan si vale la pena correr los potenciales riesgos por los beneficios involucrados. Todas estas complejidades y limitaciones son negadas por quienes se rehúsan a hacer el trabajo intelectual necesario para entender lo antes discutido y simplemente desean saber lo que las leyes o normas exigen.

\section{Justicia}

En consonancia con la pregunta previa sobre los riesgos estimados por los investigadores, también la pregunta de ¿quién debería recibir los beneficios de la investigación y quién soportar sus cargas? En México, la Ley Federal de Protección de Datos Personales en Posesión de los Particulares, ${ }^{1}$ en el artículo 21, establece:

El consentimiento podrá manifestarse de forma expresa o tácita... será expreso cuando la voluntad del titular se manifieste verbalmente, por escrito... Será tácito cuando habiéndose puesto a disposición del titular el aviso de privacidad, este no manifieste su voluntad en sentido contrario.

Y, en el artículo 22 proclama que la dispensa del consentimiento del titular para el tratamiento de sus datos personales será posible "...cuando los datos personales se sometan a un procedimiento previo de disociación".
No obstante lo promulgado por estas leyes, en la práctica persiste la controversia entre clínicos e investigadores. Algunos investigadores argumentan que con la disociación de los datos personales ocurre injusticia, porque este hecho vulnera la confidencialidad y la privacidad a las que tiene derecho el titular del contenido del expediente clínico; así como, el derecho de conocer los potenciales riesgos a los que ha sido sometido (e.g., estigmatización, pérdida de empleo, etcétera) y los beneficios a los que tiene derecho (e.g., información que resulta de la investigación, etcétera). Otros señalan que la disociación de los datos personales es injusta, porque en el proceso de recolección de datos o información de los expedientes clínicos u otras fuentes documentales, el investigador tiene acceso directo a aquellos que identifican al sujeto de investigación. $Y$, aunque en el análisis estadístico y en la publicación de resultados los datos personales se sometan a un procedimiento de disociación, el o los investigadores han tenido y tienen acceso a los datos personales, por lo que la información médica resguardada pudiera estar en riesgo de prácticas discriminatorias que afecte el acceso social de los sujetos de investigación.

De igual forma, para otros más parece injusto el consentimiento tácito aun cuando se hubiese puesto a disposición del titular el aviso de privacidad. Al respecto, la Declaración de Helsinki, pauta 22, dice: "el proyecto y el método de todo estudio en seres humanos deben describirse claramente y ser justificados en un protocolo de investigación". ${ }^{8}$ Un ejemplo especial de injusticia resulta de presentar el mencionado aviso de privacidad a individuos agobiados en muchas formas por sus enfermedades y ambientes.

¿Habría una obligación del enfermo de participar en investigaciones retrolectivas? En el plano normativo, el argumento a favor de esta obligación se deduce del hecho de que son investigaciones documentales en las que no se realiza intervención o modificación intencionada en las variables psicológicas, fisiológicas y sociales de los sujetos de investigación. ${ }^{6}$ En el plano que atiende los hechos de la conducta y las creencias morales, el argumento que respalda esta obligación se deduce de los posibles beneficios con los que la investigación podría favorecerlo y del hecho de que el enfermo puede gozar de la salud gracias a los resultados de las investigaciones que se sirvieron de otros seres humanos; por ende, a primera vista todos tendríamos el deber de participar en la contribución de tal conocimiento. 
La respuesta afirmativa es comprensible y aceptable, pero finalmente lo que cuenta no es la cantidad de beneficio sino quiénes se beneficiarán y cómo se conseguirá el acceso a la información de los expedientes clínicos para la generación de nuevo conocimiento que justifique infringir los derechos del enfermo a la autodeterminación, la intimidad y la confidencialidad de la información que ha confiado a su médico.

El investigador tiene estudios que el enfermo no tiene, de ahí que el primero tenga la firme creencia de estar en posición de saber qué es lo mejor (el bienestar social y científico) y de decidir; sin embargo, pudiera ser que los intereses de la investigación no concuerden con los intereses o creencias de los enfermos como sujetos de investigación. Cuando ambos partidos se enfrentan, ¿qué sistema de valores debiera predominar?

También pudiera ser que en una investigación retrolectiva inicialmente catalogada sin riesgo, el análisis de datos siga caminos inesperados y que resultados en un principio imprevisibles aparezcan en fases tardías. Esto compele las preguntas de si el progreso científico y social es lo único que importa en la investigación retrolectiva; y de si, al omitir el consentimiento informado o suponer el consentimiento tácito de los sujetos de investigación, las cargas y beneficios de una investigación son justamente distribuidas.

\section{Cuando la investigación científica y la ética se encuentran}

Es importante reconocer tres partes interconectadas de la infraestructura de la investigación. ${ }^{10}$ Una es la ciencia como tal, en la que se incluyen los métodos y las técnicas usadas para la generación de información; y los hechos y las teorías que conforman la actual forma de entender el mundo. Los múltiples abusos $^{11}$ que históricamente se han cometido en nombre del "progreso de la ciencia" hacen evidente la tensión existencial que el investigador enfrenta y que lo puede desconectar de la ética y moral de la medicina y la investigación científica.

La otra radica en el hecho de que un investigador es un individuo en relación con los sujetos de investigación, la sociedad y la responsabilidad consecuente. Los investigadores están inmersos en una sociedad, por lo que el trabajo científico que realizan tiene que ser producto de negociaciones sociales (autoridad, poder, amistad). Un caso real para ilustrar: en la hoja actual de consentimiento informado para la donación (de sangre) en un banco central de sangre del país, al término de la descripción de los beneficios, riesgos y procedimientos aparece una única línea que textualmente dice:

Doy mi consentimiento por propia voluntad y a título gratuito para donar sangre y acepto que la sangre donada o sus fracciones puedan ser distribuidas y utilizadas para el o los pacientes que la requieran y en los casos necesarios para fines de investigación.

Esto se hace sin especificar al donador los derechos inalienables que, como sujeto de investigación, tiene sobre el uso de los materiales donados ni la manera en que los investigadores protegerán sus datos personales (identidad) y sus datos sensibles (privacidad) durante la investigación y después de ella.

Finalmente está la parte representada por las acciones del investigador como individuo y su conciencia ética; es decir, su carácter, que incluye la forma de pensar y ser a la luz de las emociones que siente, de las expectativas, de los valores e intereses que lo motivan, de la manera como todo esto influye en sus juicios y decisiones, y de interesarse o cuestionarse las razones de sus propios actos.

En suma, es cierto que la investigación retrolectiva es necesaria porque representa un recurso importante para muchas actividades de salud pública (desde la prevención de enfermedades hasta la asignación de recursos) y para efectuar predicciones de salud y enfermedad en enfermos individuales. La contrariedad surge cuando se ignora que debajo de los mencionados problemas visibles de justicia, no maleficencia y respeto a la autonomía yacen estructuras sociales y modelos mentales de los individuos que conforman la comunidad científica. Al desconocerlos, los investigadores se condenan a continuar representando viejos patrones de abuso; o bien, a promover una rígida aplicación de la política de consentimiento o anonimato en perjuicio de la investigación retrolectiva en términos de generación de conocimiento y de tiempo.

\section{Conclusión}

El acceso a datos personales y sensibles de los enfermos con fines de investigación retrolectiva sin consentimiento de los sujetos de investigación o simplemente con el aviso de privacidad en el expediente clínico es importante para el progreso de la ciencia médica, pero tiene implicaciones éticas en la medida en que los investigadores no cumplan con las expectativas de racionalidad y responsabilidad personal que tienen ante la sociedad. 


\section{Conflicto de intereses}

Ninguno.

\section{Financiamiento}

Ninguno.

\section{Responsabilidades éticas}

Protección de personas y animales. Los autores declaran que para esta investigación no realizaron experimentos en seres humanos ni en animales.

Confidencialidad de los datos. Los autores declaran que en este artículo no aparecen datos de pacientes.

Derecho a la privacidad y consentimiento informado. Los autores declaran que en este artículo no aparecen datos de pacientes.

\section{Bibliografía}

1. Ley Federal de Protección de Datos Personales en Posesión de los Particulares. México: Diario Oficial de la Federación. 2010 Jul 05

2. Ley General de Protección de Datos Personales en Posesión de Sujetos Obligados. México: Diario Oficial de la Federación; 2017 Ene 26.

3. Norma Oficial Mexicana NOM-004-SSA3-2012, del expediente clínico. México: Diario Oficial de la Federación; 2012 Oct 12.

4. Norma Oficial Mexicana NOM-024-SSA3-2012, sistemas de información de registro electrónico para la salud. Intercambio de información en salud. México: Diario Oficial de la Federación; 2012 Nov 30.

5. Feinstein AR. Clinical epidemiology: the architecture of clinical research. EE. UU.: Saunders; 1985.

6. Reglamento de la Ley General de Salud en Materia de Investigación para la Salud. México: Diario Oficial de la Federación; 2014.

7. Beauchamp TL, Childress JF. Principles of biomedical ethics. EE. UU.: Oxford University Press; 2013

8. Asociación Médica Mundial [Internet]. Declaración de Helsinki. Principios éticos para las investigaciones médicas en seres humanos. 64a Asamblea General de la AMM; 2020.

9. International ethical guidelines for health-related research involving humans. Suiza: Council for International Organizations of Medical Sciences; 2016.

10. Lonergan SJB: Cognitional structure. En: Crowe FE, Doran RM, editores. Collected works of Bernard Lonergan. Canadá: University of Toronto Press; 1967.

11. Alexander L. Medical science under dictatorship. N England J Med. 1949;241:39-47 